\title{
Atuação do farmacêutico clínico frente a pandemia da COVID-19
}

\author{
The clinical pharmacist's performance against the COVID-19 pandemic
}

El papel del farmacéutico clínico en la pandemia del COVID-19

Recebido: 06/09/2021 | Revisado: 12/09/2021 | Aceito: 17/09/2021 | Publicado: 18/09/2021

\author{
Daniella Cavalcante da Silva \\ ORCID: https://orcid.org/0000-0002-1883-405X \\ Faculdade Integrada Carajás, Brasil \\ E-mail: daniellacavalcante10@gmail.com \\ Nathana Costa Alves \\ ORCID: https://orcid.org/0000-0002-5971-402X \\ Faculdade Integrada Carajás, Brasil \\ E-mail: nathanaalvesfarmacia@gmail.com \\ Carolinne de Oliveira Marquez \\ ORCID: https://orcid.org/0000-0001-6556-5094 \\ Faculdade Integrada Carajás, Brasil \\ E-mail: carolzinhaoliveiramarquez@yahoo.com.br
}

\begin{abstract}
Resumo
Nos dias atuais o farmacêutico assume um papel de grande importância, tendo ações voltadas para o uso racional de medicamentos, com a pandemia, gerencia a farmácia hospitalar voltadas para o combate ao COVID-19 e nas farmácias comunitárias prestando atenção farmacêutica. O estudo tem como objetivo descrever a atuação do profissional durante a pandemia. O presente estudo trata-se de uma revisão integrativa, sendo fundamentada na pesquisa bibliográfica estabelecida por meio de artigos publicados sobre a atuação do farmacêutico clínico frente a COVID-19. A coleta foi realizada no período de fevereiro e março de 2021, onde foram investigados estudos publicados nas bases de dados: Science Direct, PubMed e MEDLINE por meio dos Periódicos Capes. A atuação do farmacêutico no combate ao coronavírus tem sido fundamental para orientar pacientes com comorbidades que buscam informações sobre os medicamentos no processo patológico da COVID-19, fornecimento e controle dos estoques de materiais médico-hospitalares, nas atividades assistenciais clínicas, lidando com o uso seguro e racional de medicamentos, prevenindo erros relacionados a medicamentos e o cuidado com a estabilidade das drogas, seja após diluição ou reconstituição, foi fundamental para a economia e o aproveitamento de recursos escassos. Por estarmos passando por momentos difíceis, o farmacêutico está atuando frente a essa pandemia tornando-se um profissional essencial, uma vez que, possui total respaldo para integrar as equipes de saúde de frente, desenvolvendo toda a sua expertise a respeito dos medicamentos, doses, efeitos adversos e interações medicamentosas, dando suporte a outros profissionais e orientando os pacientes com a atenção farmacêutica.
\end{abstract}

Palavras-chave: Farmacêutico clínico; Medicamentos; Pandemia; COVID-19.

\begin{abstract}
Abstratc
Nowadays, the pharmacist plays a very important role, taking actions aimed at the rational use of medicines, with the pandemic, he manages the hospital pharmacy aimed at combating COVID-19 and in community pharmacies providing pharmaceutical care. The study aims to describe the professional's performance during the pandemic. The present study is an integrative review, based on bibliographic research established through articles published on the role of clinical pharmacists in the face of COVID-19. Data collection was carried out between February and March 2021, where studies published in the following databases were investigated: Science Direct, PubMed and MEDLINE through Capes Periodicals. The role of the pharmacist in combating the coronavirus has been fundamental in guiding patients with comorbidities who seek information about drugs in the pathological process of COVID-19, supply and control of stocks of medical and hospital materials, in clinical care activities, dealing with their use safe and rational medications, preventing errors related to medications and taking care of the stability of drugs, whether after dilution or reconstitution, was fundamental for the economy and the use of scarce resources. As we are going through difficult times, the pharmacist is acting in the face of this pandemic, becoming an essential professional, since he has full support to integrate the health teams at the front, developing all his expertise regarding medicines, doses, adverse effects and drug interactions, supporting other professionals and guiding patients with pharmaceutical care.
\end{abstract}

Keywords: Clinical Pharmacist; Medicines; Pandemic; COVID-19.

\section{Resumen}

En la actualidad, el farmacéutico juega un papel muy importante, realizando acciones encaminadas al uso racional de los medicamentos, con la pandemia, gestiona la farmacia hospitalaria destinada a combatir el COVID-19 y en las 
farmacias comunitarias que brindan atención farmacéutica. El estudio tiene como objetivo describir el desempeño del profesional durante la pandemia. El presente estudio es una revisión integradora, basada en la investigación bibliográfica establecida a través de artículos publicados sobre el papel de los farmacéuticos clínicos frente al COVID-19. La recolección de datos se realizó entre febrero y marzo de 2021, donde se investigaron los estudios publicados en las siguientes bases de datos: Science Direct, PubMed y MEDLINE a través de Capes Periodicals. El papel del farmacéutico en el combate al coronavirus ha sido fundamental para orientar a los pacientes con comorbilidades que buscan información sobre medicamentos en el proceso patológico del COVID-19, suministro y control de stocks de material médico-hospitalario, en las actividades de atención clínica, atendiendo a sus necesidades. utilizar medicamentos seguros y racionales, prevenir errores relacionados con los medicamentos y cuidar la estabilidad de los medicamentos, ya sea después de su dilución o reconstitución, fue fundamental para la economía y el uso de recursos escasos. Ante los momentos difíciles que atravesamos, el farmacéutico está actuando frente a esta pandemia, convirtiéndose en un profesional imprescindible, ya que cuenta con pleno apoyo para integrar los equipos de salud en el frente, desarrollando toda su experiencia en cuanto a medicamentos, dosis, efectos adversos y interacciones farmacológicas, apoyando a otros profesionales y guiando a los pacientes con la atención farmacéutica.

Palabras clave: Farmacéutico clínico; Medicamentos; Pandemia; COVID-19.

\section{Introdução}

Os serviços realizados por farmacêuticos em farmácias comunitárias, além de oferecer tratamento e segurança aos pacientes, transformou o ambiente em um espaço que cuida da saúde da população, com a presença de profissionais qualificados.

Para Kretchy et al. (2020) os profissionais farmacêuticos tem uma grande importância neste momento de crise atual, na gestão da farmácia hospitalar no combate a COVID-19, como também nas farmácias comunitárias prestando assistência. O farmacêutico tem a responsabilidade de informar sobre o uso racional de medicamentos, pelo motivo que muitas pessoas estão comprando e consumindo na prevenção da COVID-19, no caso a cloroquina e hidroxicloroquina.

Em Wuhan na China em 2019, surgiram informações de uma pneumonia séria, mas não definida. Com estudos laboratoriais detectaram um vírus chamado coronavírus e causa infecções no sistema respiratório (Lythgoe; Middleton, 2020).

A Organização Mundial de Saúde (OMS) declarou este cenário como emergência de saúde pública no dia 30 de janeiro, pelo motivo que os casos aumentaram rápido e espalhou-se por muitos países (Brasil, 2020).

Segundo Zheng et al. (2020) a origem deste vírus ainda é desconhecida, porém há relatos que relacionam os primeiros casos com um mercado de frutos do mar em Wuhan onde são comercializados animais selvagens. Devido ao avanço da doença em diversos países em todo o mundo, em 11 de março de 2020 a OMS declara o novo coronavírus como uma pandemia, uma vez que os casos confirmados aumentaram drasticamente em muitos países com casos graves levando a óbito.

Tay et al. (2020) descrevem os sintomas principais que são: febre, tosse seca e dispnéia. Alguns casos também ocasionam cefaléia, diarréia e mialgias. Geralmente o período de incubação vai de 2 a 14 dias após a infecção. O vírus se espalhou por gotículas respiratórias com tosse ou espirro e também ocorreu a contaminação por objetos infectados quando tocados e levados a boca, nariz e olhos.

Desse modo, deparamo-nos com uma questão a ser questionada: De que maneira a atuação da equipe de farmácia auxilia no combate a pandemia da COVID-19?

Assim, este artigo tem o objetivo de analisar a atuação da equipe de farmácia no auxílio ao tratamento da COVID-19, com enfoque nas principais ações exercidas por este profissional da saúde no Brasil.

\section{Metodologia}

O presente estudo trata-se de uma revisão integrativa sendo fundamentada na pesquisa bibliográfica, método que agrupa a produção científica relevante acerca de um tema preestabelecido, ofertando acesso rápido e sintetizado aos resultados científicos de maior acuidade para a área estudada, estabelecida por meio de artigos publicados sobre a atuação do 
farmacêutico clínico frente a COVID-19. E para consolidação da mesma seguiu-se as seguintes etapas: escolha do tema, levantamento bibliográfico preliminar, formulação do problema, busca de fontes, leitura do material, organização lógica do assunto e por fim redação do texto.

A coleta foi realizada no período de fevereiro e março de 2021, onde foram investigados estudos publicados nas bases de dados: Science Direct, PubMed e MEDLINE por meio dos Periódicos Capes, empregando-se os descritores farmacêutico clínico, medicamento, pandemia e COVID referente ao período de 2016 a 2021.

Foram incluídos artigos completos em português e inglês de 2016 e 2021 que tratavam da temática em questão e que estavam disponibilizados eletronicamente na íntegra e excluídos os artigos publicados fora do período de 2016 a 2021 e que não estivessem condizentes com o tema abordado.

Ao iniciar a pesquisa foram encontrados 80 artigos relacionados sobre a atuação do farmacêutico clínico frente a COVID-19 dos quais 50 apresentavam repetições nas bases de dados, ficando 30 artigos para a leitura. Durante o processo de análise 20 artigos foram eliminados por não corresponderem as principais adaptações dos critérios estabelecidos, resultando em 10 artigos selecionados de acordo com a abordagem e objetividade do tema na elaboração do artigo. O fluxograma da Figura 1 demostra de forma transparente todas as metodologias de busca pelas pesquisas escolhidas para a confecção da respectiva revisão.

Figura 1 - Fluxograma da pesquisa bibliográfica.

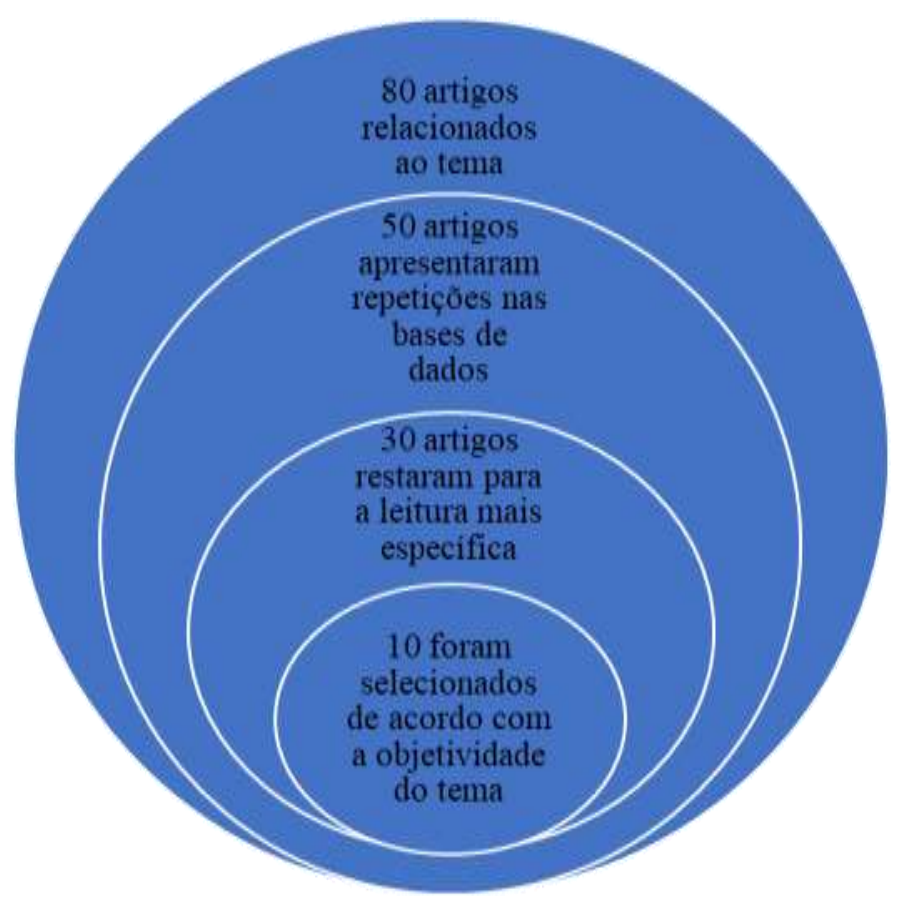

Fonte: Autoras, Redenção - PA, 2021.

\section{Referencial Teórico}

\subsection{Atribuições do farmacêutico na pandemia}

Segundo Zheng et al. (2020) o farmacêutico hoje tem uma importância grande nesse período de pandemia, decorrente do isolamento que as pessoas estão passando e por não poderem ir ao hospital (lugar onde tem mais pacientes internados pelo vírus). Nas farmácias, os profissionais tem a missão de orientar e direcionar o uso adequado dos medicamentos. 
Os cuidados que o farmacêutico tem que ter na comunidade são os fornecimentos corretos dos medicamentos, prevenindo no controle da Covid-19 e tirando todas as dúvidas referente ao contágio, sintomas e a automedicação (KRETCHY et al., 2020).

Codogan e Hugues (2020) enfatiza que os indivíduos que tem comorbidades crônicas precisam de mais atenção, por precisarem continuar com seus tratamentos e são mais propícios a se contagiar com SARS-CoV2. Os farmacêuticos devem orientá-los com sua farmacologia, atentando aos horários e quantidades corretas.

Conforme Song et al. (2020), o farmacêutico clínico atua dentro de hospitais e seu trabalho é muito relevante, pois o profissional irá fiscalizar desde o abastecimento dos medicamentos da farmácia hospitalar e até onde chega ao paciente internado. Diante disso, estudaram as possibilidades de como cuidar desses pacientes hospitalizados centralizando-os num determinado local para tratarem melhor e com recursos apropriados.

Determinados serviços de assistência farmacêutica se destacaram na pandemia. A função do farmacêutico é de assegurar o uso correto dos medicamentos que são utilizados nos hospitais, uma vez que não tem comprovação de medicamento eficaz para a Covid-19. Os medicamentos que já estão usando para outras patologias, estão sendo administrados como uso off-label (LIU et al., 2020).

\subsection{O Uso razoável de medicamentos}

O Conselho Federal de Farmácia na resolução 586/2013, descreve que o farmacêutico frente a Covid-19, se encontra apto para exercer seu trabalho promovendo e orientando o uso adequado de medicamentos, com testes simples, notificando suspeitos e encaminhando para o hospital para deteç̧ão correta do diagnóstico. As pessoas que procuram as farmácias vêm crescendo muito, quando sentem algum sintoma parecido da Covid-19 (CFF, 2020).

A cloroquina é um medicamento antimalárico e vem sendo usado pela população no tratamento da infecção do novo coronavírus (TOURET, LAMBALLERIE, 2020).

"Esse medicamento inibe a fosforilação terminal da Enzima Conversora de Angiotensina II (ACE2), e a hidroxicloroquina eleva o pH nos endossomos envolvidos na entrada das células virais" (MCKEE et al., 2020).

Touret e Lamballerie (2020) citam que os efeitos da dose terapêutica e a tóxica são parecidas ocasionando distúrbios cardiovasculares que pode levar o paciente a óbito. Os efeitos colaterais crescem no intervalo QT, taquicardia e fibrilação ventricular. Portanto o uso da cloroquina e hidroxicloroquina devem ser usados no ambiente hospitalar, prescritos por um médico.

O uso desses medicamentos não apresenta nenhum benefício para o paciente diagnosticado com Covid-19 e sim apresentaram arritmias ventriculas e risco de morte, estudo que foi publicado na revista The Lancet (MEHRA et al., 2020).

Rossignol (2016) descreve a nitazoxanida como um agente antiviral e antiparasitário de amplo espectro em desenvolvimento para o tratamento da gripe e outras infecções respiratórias virais como também para infecções intestinais causados por Cruptosporidium parvum, espécie de parasita que causa a criptosporidíase.

De acordo com Ying et al. (2020) muitos países estão usando esses medicamentos, por não ter uma confirmação de eficácia e segurança, diante disso, o farmacêutico contribui no acompanhamento desses pacientes, decorrentes aos efeitos adversos desses medicamentos, controlando as doses conforme a situação de cada paciente em parceria com a equipe médica.

\subsection{Serviços farmacêuticos}

Conforme Araújo, Grassi e Teixeira (2020) qualquer pessoa pode contrair o vírus e apresentar problemas respiratórios, onde não tem idade, sexo ou cor. No entanto, as pessoas mais propensas são de meia-idade e idosos, sendo do 
grupo de risco. Na China a maioria dos infectados variaram de 49 a 56 anos. A mortalidade com taxa de $8 \%$ a $15 \%$ entre pessoas de 70 a 80 anos. No momento as crianças são assintomáticas.

Ferreira et al. (2019) afirmam que a população precisa de ensinamentos para cuidar melhor da saúde, seja de maneira individual ou coletiva, e é preciso alcançar as deficiências enfrentadas pela população durante um tratamento farmacológico ou não farmacológico, enfatizando para mudanças de hábitos alimentares e físicos. Faz-se necessário orientação sobre adesão, observando sempre o ambiente em que as pessoas residem, condição financeira e social a fim de evitar a adoção de medidas infundadas, impossíveis de serem adotadas naquela localidade.

Pesquisar e conhecer o perfil da população possibilita a implementação de serviços farmacêuticos conforme a necessidade, permite orientar com eficiência sobre a adesão ao tratamento com medicamentos ou simplesmente com alterações comportamentais e alimentares, descobrir a problemática vivenciada por cada paciente é a chave do sucesso para uma aceitação e fidelização por parte daquela população assistida (SILVA et al., 2018).

O uso racional de medicamentos tornou-se uma preocupação constante dos órgãos de saúde. Logo, o conhecimento em atenção farmacêutica coloca o farmacêutico em evidência para realizar atendimento e explicação a população para o uso correto de medicamentos. Essa iniciativa dentro das farmácias proporciona fidelização de clientes, proporcionando retorno financeiro satisfatório. Sendo a farmácia um estabelecimento de saúde, é imprescindível a presença de um profissional com conhecimento científico para coordenar as atividades, já que a maioria dos atendentes possuem apenas o conhecimento prático, insuficiente para um atendimento ideal (OLIVEIRA et al., 2017).

Correr (2019) descreve alguns serviços: o rastreamento em saúde, atende pessoas assintomáticas, visando detectar ou evitar possíveis doenças. Já o manejo de problemas de saúde autolimitados, quando o paciente procura um farmacêutico para relatar algo que o incomoda, nesse momento acontece a consulta, onde poderá ser indicado medicamento que não exige receita médica ou direcionamento para um profissional especializado. Educação em saúde visa orientar o paciente quanto ao uso de medicamentos e medidas capazes de reduzir o fluxo hospitalar.

O serviço de conciliação de medicamentos é direcionado aos pacientes que receberam alta hospitalar, facilita a adesão e evita internamentos. A revisão da farmacoterapia confere todos os medicamentos usados pelo paciente e detecta possíveis problemas relacionados ao uso. O acompanhamento farmacoterapêutico permite uma aproximação maior entre paciente e farmacêutico, que poderá ser duradouro. Gestão da condição de saúde, o profissional foca em uma doença específica, auxiliando o paciente a cuidar melhor de uma enfermidade (CORRER, 2019).

A Atenção Farmacêutica facilita a entrada do farmacêutico na equipe multidisciplinar, agrega valores à profissão onde na maioria das vezes é observada com surpresa por parte das pessoas ao visualizarem as novas atividades dos profissionais farmacêuticos. Já que o trabalho dos farmacêuticos poderá trazer benefícios aos pacientes, observa-se que a implantação de serviços farmacêuticos soma positivamente, trazendo bons resultados junto a população assistida (BRASIL, 2016).

\section{Resultados}

Dos 10 artigos selecionados, 5 são revisões bibliográficas e 5 de revisões descritivas/experimentais. O perfil e características dos estudos estão apresentados na Tabela 1. 
Tabela 1- Principais estudos sobre o papel do farmacêutico frente a COVID-19 encontrados a partir da busca bibliográfica.

\begin{tabular}{|c|c|c|c|}
\hline ARTIGO & OBJETIVO & $\begin{array}{l}\text { DESENHO DO } \\
\text { ESTUDO } \\
\end{array}$ & CONSIDERAÇÕES FINAIS \\
\hline Ying et al. (2020). & $\begin{array}{c}\text { Apresentar a importância da } \\
\text { atuação do profissional } \\
\text { farmacêutico frente a pandemia } \\
\text { do COVID-19. }\end{array}$ & Revisão Bibliográfica. & $\begin{array}{l}\text { O farmacêutico comunitário e clínico que estão } \\
\text { atuando frente a essa pandemia têm se tornado um } \\
\text { profissional essencial, uma vez que, possui total } \\
\text { respaldo para integrar as equipes de saúde de } \\
\text { frente, desenvolvendo toda a sua expertise a } \\
\text { respeito dos medicamentos, doses, efeitos adversos } \\
\text { e interações medicamentosas, dando suporte a } \\
\text { outros profissionais e orientando os pacientes com } \\
\text { a atenção farmacêutica. }\end{array}$ \\
\hline CHLO (2020). & $\begin{array}{c}\text { Analisar o exercício da } \\
\text { atividade farmacêutica visando } \\
\text { o cidadão em geral e o doente } \\
\text { em particular durante a } \\
\text { pandemia COVID-19. }\end{array}$ & $\begin{array}{c}\text { Revisão } \\
\text { Descritiva/Experimental. }\end{array}$ & $\begin{array}{l}\text { Ao nível dos Serviços Farmacêuticos Hospitalar, a } \\
\text { reestruturação interna para dar resposta às novas } \\
\text { demandas determinadas pela COVID-19 teve de ser } \\
\text { feita simultaneamente cuidando das pessoas e } \\
\text { trabalhando em equipa, focando na identificação } \\
\text { das soluções mais adequadas em cada momento } \\
\text { para os diferentes contextos da dinâmica hospitalar, } \\
\text { incluindo para os doentes em regime de } \\
\text { internamento ou ambulatório. }\end{array}$ \\
\hline $\begin{array}{c}\text { Mendonça e } \\
\text { Rossoni (2020). }\end{array}$ & $\begin{array}{l}\text { Buscar correlacionar as novas } \\
\text { demandas impetradas aos } \\
\text { estabelecimentos farmacêuticos } \\
\text { com os Objetivos do } \\
\text { Desenvolvimento Sustentável } \\
\text { (ODS) preconizados pela } \\
\text { Agenda } 2030 \text { da Organização } \\
\text { das Nações Unidas. }\end{array}$ & Revisão Bibliográfica & $\begin{array}{l}\text { Após o estudo dos ODS promulgados pela Agenda } \\
\text { 2030, observa-se que o papel socioambiental do } \\
\text { farmacêutico vem ao encontro de alguns dos } \\
\text { objetivos e metas estabelecidos pela ONU em } \\
\text { aspectos socioeconômicos ambientais, de modo a } \\
\text { contribuir em áreas de importância crucial para a } \\
\text { humanidade e para o planeta neste momento de } \\
\text { pandemia de COVID-19. }\end{array}$ \\
\hline Silveira (2019). & $\begin{array}{c}\text { Analisar a execução do ciclo da } \\
\text { assistência farmacêutica, frente } \\
\text { a casos graves da COVID-19, } \\
\text { em um hospital de referência no } \\
\text { município de Fortaleza. }\end{array}$ & Revisão Experimental. & $\begin{array}{l}\text { Evidencia-se que a adequação e norteamento das } \\
\text { ações da assistência farmacêutica em meio a } \\
\text { pandemia declarada da COVID-19 se torna eficaz } \\
\text { para compreensão dos procedimentos a serem } \\
\text { realizados em meio a uma situação tão complexa } \\
\text { como esta. Cabe ao farmacêutico junto a equipe } \\
\text { multiprofissional em saúde acompanhar a utilização } \\
\text { destes medicamentos e a evolução clínica dos } \\
\text { pacientes visto que o uso off label de medicamentos } \\
\text { pode gerar sérios agravos a saúde dos indivíduos } \\
\text { que já estão internados em estado grave e situação } \\
\text { crítica. São necessários estudos clínicos mais } \\
\text { robustos para comprovação da ação da cloroquina e } \\
\text { hidroxicloroquina frente a COVID-19. }\end{array}$ \\
\hline $\begin{array}{l}\text { Araújo, Grassi e } \\
\text { Teixeira (2020). }\end{array}$ & $\begin{array}{l}\text { Descrever os aspectos } \\
\text { farmacológicos, terapias } \\
\text { propostas, protocolos } \\
\text { terapêuticos e cuidados } \\
\text { farmacêuticos no contexto da } \\
\text { Covid-19. }\end{array}$ & Revisão Bibliográfica. & $\begin{array}{l}\text { Diante de tantas terapêuticas, surgem expectativas } \\
\text { para que a situação pandêmica que o mundo se } \\
\text { encontra possa ser revertida e que tudo comece aos } \\
\text { poucos a se normalizar. Existe uma necessidade } \\
\text { urgente de entender melhor o vírus e desenvolver } \\
\text { meios farmacológicos para controlar a propagação, } \\
\text { para isso os novos tratamentos para a Covid-19 } \\
\text { devem não apenas ser seguros e eficazes, mas } \\
\text { também baratos e prontamente disponíveis. }\end{array}$ \\
\hline $\begin{array}{c}\text { Le, Toscani e } \\
\text { Colaizzi (2020). }\end{array}$ & $\begin{array}{l}\text { Apresentar como o serviço de } \\
\text { Telecuidado Farmacêutico foi } \\
\text { estruturado e ofertado no } \\
\text { combate à COVID-19 como } \\
\text { uma estratégia emergente. }\end{array}$ & $\begin{array}{c}\text { Revisão } \\
\text { Descritiva/Experimental. }\end{array}$ & $\begin{array}{l}\text { Para a teoria, fica mais um registro da importância } \\
\text { de reconhecer a estratégia emergente como um } \\
\text { desenho estratégico adequado a contextos instáveis } \\
\text { e a implementação de serviços no âmbito da gestão } \\
\text { em saúde. A concepção fundamentada na lógica do } \\
\text { plano deliberado como uma antecipação da } \\
\text { execução tem cada vez menos sentido em um } \\
\text { mundo dinâmico, no qual a sociedade demanda } \\
\text { novas soluções para novos problemas. A moldagem } \\
\text { da estratégia emergente tem a capacidade de } \\
\text { responder a transformações de nosso tempo de }\end{array}$ \\
\hline
\end{tabular}




\begin{tabular}{|c|c|c|c|}
\hline & & & $\begin{array}{l}\text { maneira mais efetiva, trazendo benefícios imediatos } \\
\text { para os usuários do sistema de saúde. }\end{array}$ \\
\hline SESPR, 2020. & $\begin{array}{c}\text { Apresentar o relato de } \\
\text { experiência do planejamento, da } \\
\text { execução e dos resultados das } \\
\text { ações adotadas pela Assistência } \\
\text { Farmacêutica da SESA/PR } \\
\text { voltadas à redução do potencial } \\
\text { de contaminação de usuários e } \\
\text { servidores pelo novo } \\
\text { coronavírus, no âmbito das } \\
\text { farmácias e centrais de } \\
\text { abastecimento farmacêutico das } \\
\text { Regionais de Saúde. }\end{array}$ & Revisão Descritiva. & $\begin{array}{l}\text { As medidas foram efetivas na redução da } \\
\text { circulação de pessoas e aumento do distanciamento } \\
\text { social, diminuindo assim o risco de contágio pelo } \\
\text { novo coronavírus tanto dos usuários quanto das } \\
\text { equipes da assistência farmacêutica. }\end{array}$ \\
\hline Silva, (2018). & $\begin{array}{c}\text { Analisar as atuações do oficial } \\
\text { farmacêutico no enfrentamento } \\
\text { da pandemia de COVID-19, a } \\
\text { fim de evidenciar a importância } \\
\text { deste militar. }\end{array}$ & Revisão Bibliográfica. & $\begin{array}{c}\text { Conclui-se que estes oficiais vêm atuando como } \\
\text { elementos fundamentais nesse combate em diversos } \\
\text { campos de batalha cotidiana, como na área } \\
\text { diagnóstica realizando testes laboratoriais. a } \\
\text { atuação desses profissionais na produção de } \\
\text { conhecimento científico, elevando o prestígio da } \\
\text { Força Terrestre, além de contribuir para a } \\
\text { valorização destes oficiais junto à comunidade } \\
\text { acadêmica e à sociedade. }\end{array}$ \\
\hline $\begin{array}{l}\text { Oliveira, Szabo e } \\
\text { Bastos (2017). }\end{array}$ & $\begin{array}{c}\text { Analisar os serviços } \\
\text { farmacêuticos, seu efeito } \\
\text { perante a sociedade e os } \\
\text { resultados obtidos pelas } \\
\text { empresas que os oferecem, além } \\
\text { de fortalecer o papel do } \\
\text { profissional farmacêutico como } \\
\text { gestor. }\end{array}$ & Revisão Bibliográfica. & $\begin{array}{l}\text { Os serviços prestados nas farmácias e drogarias } \\
\text { além de trazer qualidade de vida para os pacientes, } \\
\text { fortalece junto à comunidade o profissional } \\
\text { farmacêutico. Além disso, percebe-se que a } \\
\text { implementação dos serviços em farmácias } \\
\text { comunitárias esbarra em dificuldades financeiras e } \\
\text { falta de tempo dos farmacêuticos que tem que se } \\
\text { dividir entre o atendimento no balcão e a consulta. }\end{array}$ \\
\hline $\begin{array}{l}\text { Lima et al. } \\
\quad(2020)\end{array}$ & $\begin{array}{l}\text { Identificar os desafios e as } \\
\text { respostas da Secretaria } \\
\text { Municipal de Saúde de Franco } \\
\text { da Rocha, no âmbito da } \\
\text { Assistência Farmacêutica, } \\
\text { frente à pandemia. }\end{array}$ & $\begin{array}{c}\text { Revisão } \\
\text { Descritiva/Exploratória. }\end{array}$ & $\begin{array}{l}\text { Os resultados mostram que a maioria dos } \\
\text { entrevistados está satisfeita com as orientações } \\
\text { fornecidas e ações efetuadas pela gestão dos } \\
\text { serviços de saúde para garantir a higienização e } \\
\text { segurança de todos nas UBS. Quanto à } \\
\text { disponibilidade de EPI, diversas denúncias de } \\
\text { profissionais de saúde foram efetuadas no país, mas } \\
\text { o município de Franco da Rocha demonstrou } \\
\text { planejamento e capacidade de resposta efetivos, } \\
\text { conforme relato dos entrevistados. }\end{array}$ \\
\hline
\end{tabular}

Fonte: Próprias autoras, Redenção-PA, 2021.

\section{Discussão}

Durante essa pandemia o profissional farmacêutico clínico merece atenção, visto que é fundamental seus serviços desde o abastecimento de medicamentos da farmácia hospitalar à atenção com os pacientes internados.

Ying et al. (2020) relata que no Hospital da Universidade de Jilin (THJU) - China foi elaborada uma estratégia com os farmacêuticos. Dividiram-se em equipes, onde a primeira foram farmacêuticos experientes no combate a pandemia e a segunda formado por farmacêuticos clínicos e analistas clínicos. Neste período foram observados a saúde mental dos farmacêuticos.

No Centro Hospitalar de Lisboa Ocidental os serviços farmacêuticos hospitalar (SFH) concebe uma estrutura importante aos cuidados de saúde no âmbito hospitalar, pertencendo ao grupo de serviços de apoio à ação médica. O funcionamento integra uma equipe farmacêutica, assistentes técnicos (AT), assistentes operacionais (AO) e técnicos superiores de diagnóstico e terapêutica (Chlo, 2020). 
Mendonça e Rossoni (2020) enfatiza o papel do Conselho Regional de Farmácia de Minas Gerais com a responsabilidade de prestar apoio técnico aos farmacêuticos, forneceu o acesso livre e gratuito de materiais técnicos sobre o descarte e a logística oposta de medicamentos e orientações sobre gerenciamento dos resíduos sólidos.

Conforme a explanação de Silveira (2019) as atividades clínicas do farmacêutico que podem ser agregados neste serviço ressalta o cuidado e solução de problemas com medicamentos, diminuindo o tempo do paciente no hospital e evitando a mortalidade, reduzindo os custos hospitalares e os recursos derivado do setor público e as atividades de farmacoepidemiologia e farmacovigilância dispõe como objetivo a geração de informação na qualificação da assistência farmacêutica no SUS.

De acordo com Araújo, Grassi e Teixeira (2020), o farmacêutico tem a liberação para fazer os testes rápido na drogaria e o resultado sendo positivo, ele pode realizar a anamnese do paciente, examinar os sinais vitais como também o encaminhar para o hospital ou UPA (Unidade de Pronto Atendimento) mais próximo.

Complementando o estudo, Le, Toscani e Collaizzi (2020) descreve a evolução tecnológica chamada "telefarmácia" que ajudou nos serviços farmacêuticos e com isso diminuiu os problemas relacionados aos medicamentos. Os serviços de telefarmácia podem ser oferecidos por farmácias comunitárias, hospitais, ambulatórios e por casas de repouso. As vantagens incluem despesas baixa, comodidade para o paciente com dificuldade de locomoção e serviço de orientação. Esse serviço tem que ter uma ligação a um serviço presencial e ser efetuado por um farmacêutico com foco no indivíduo.

A Secretaria de Estado da Saúde do Paraná elaborou um planejamento para diminuir o fluxo de indivíduos nas farmácias das Regionais de Saúde incluíram a ampliação de ferramenta para cadastro on line, renovação automática na continuidade do tratamento, aumento do serviço "Remédio em casa", entrega antecipada de medicamentos para dois ou três meses de tratamento e envio de mensagens com orientações para os indivíduos (Brasil, 2020).

Durante a pesquisa Silva (2018) relata que o Exército Brasileiro passou por momentos terríveis na pandemia, decorrente aos riscos de contaminação em massa. Foram escolhidas diversas equipes do Instituto de Biologia do Exército (IBEx), todas sob chefia de um oficial farmacêutico, para a coleta e realização dos exames de militares alocados em escolas de formação, institutos militares e centros de instrução.

Desta maneira, é imprescindível o reconhecimento do farmacêutico pela população brasileira e pelos órgãos públicos. Muitos empresários não valorizam o serviço que o farmacêutico desenvolve, pois desempenha várias funções, o que prejudica a atenção farmacêutica (Oliveira; Szabo; Bastos, 2017).

Lima et al. (2020) apontam numa pesquisa feita em Franco da Rocha - São Paulo, que os farmacêuticos relataram que no início da pandemia faltaram muitos medicamentos prescritos como o antiviral Tamiflu (nome comercial de oseltamivir) e do antibiótico azitromicina. No ambiente hospitalar se usavam em grande quantidade a fentanila, epinefrina (utilizados no processo de intubação de pacientes) e prednisona.

\section{Conclusão}

O mundo passa por um contágio de um vírus assustador, onde mata rápido e senão morre deixa sequelas graves. As vidas das pessoas mudaram completamente, no qual a maioria está no isolamento social e outras se prevenindo usando máscaras e álcool em gel.

Evidencia-se que a adequação e norteamento das ações da assistência farmacêutica em meio a pandemia declarada de COVID-19 se torna eficaz para compreensão dos procedimentos a serem realizados em meio a uma situação tão complexa como esta. 
Os farmacêuticos estão atuantes nessa pandemia e tem se tornado profissionais exemplares, onde tem um trabalho importante de interação com as equipes de saúde e desenvolvem a indicação a respeito dos medicamentos, efeitos adversos, doses, internações medicamentosas e dando suporte a outros profissionais com atenção farmacêutica.

Depois dessa pandemia, o profissional farmacêutico terá mais clareza e mais competência referente a esses momentos turbulentos, ao qual adquiriu muita experiência e motivação e a atenção farmacêutica será mais defendida e valorizada. Estamos passando ainda por essa pandemia e os dados pesquisados estão sendo atualizados diariamente, diante disso surgindo alterações.

Muitas pesquisas ainda estão em andamento e novos resultados surgiram referente ao novo vírus, sendo assim a equipe de farmácia continua vivenciando novos acontecimentos a cada dia com o surgimento da variante que é mais forte. Portanto, estamos ainda passando por essa pandemia, estamos sendo vacinados e ainda assim, não tem data para acabar.

Para trabalhos futuros sugerimos que enfatizem a atuação de farmacêuticos clínicos como parte de uma equipe multidisciplinar, evidenciando desafios, descobertas e aprendizados vividos durante toda a pandemia, a fim de trazer a visão bem como mostrar a importância desse profissional para a equipe de saúde.

\section{Referências}

Araujo, L. F. S., Strina, A., Grassi, M. F. R. G. \& Teixeira, M. G. (2020). Aspectos clínicos e terapêuticos da infecção da COVID-19. ARCAR FIOCRUZ, 2020. https://www.arca.fiocruz.br/bitstream/icict/40662/2/Aspectos-cl\%c3\%adnicos-e-terap\%c3\%aauticos-da-infec\%c3\%a7\%c3\%a3o-da-COVID-19-1.pdf.

Bousquat, A., Giovanella, L., Medina, M. G., Mendonça, M. H. M., Facchini, L. A. \& Tasca, R. (2020). Desafios da Atenção Básica no enfrentamento da pandemia da Covid-19 no SUS. Relatório de Pesquisa. USP, Fiocruz, UFBA, UFPEL, OPAS Brasil. Rede de Pesquisa em Atenção Primária à Saúde da Abrasco. Rio de Janeiro: Rede de Pesquisa em APS Abrasco. https://redeaps.org.br/wp-content/uploads/2020/11/Relat\%C3\%B3rioEstadual_RJ_24.10.20201.pdf.“.

Cadogan, C. A. \& Hughes, C. M. (2021). On the frontline against COVID-19: Community pharmacists' contribution during a public health crisis. Research in Social and Administrative Pharmacy, 17(1), 2032-5. https://www.sciencedirect.com/science/article/abs/pii/S1551741120302928.“.

Correr, C. (2019). Farmácia, Serviços Farmacêuticos: 7 tipos que podem ajudar sua farmácia, Curitiba, v. 1, n. 1, p.1-5. https://clinicarx.com.br/blog/7-tiposde-servicos-farmaceuticos-que-podem-ajudar-sua-farmacia/.

da Silva, A. A. (2018). No sinuoso trilho do empreendedorismo. Análise Social, 53(229 (4), 984-1009. http://analisesocial.ics.ul.pt/documentos/n229_a06.pdf.

de Albuquerque, N. L. S. (2020). Planejamento operacional durante a pandemia de COVID-19: comparação entre recomendações da Organização Mundial da Saúde e o plano de contingência nacional. Cogitare Enfermagem, 25. http://revista.farmacoterapia.pt/index.php/rpf/article/view/279.

de Farmácia, C. F. (2020). Guia para estratificação de risco de suspeitos de Covid-19 e delineamento de cuidado farmacêutico para farmácias comunitárias. Brasília: Conselho Federal de Farmácia. http://covid19.cff.org.br/wp-content/uploads/2020/05/delineamento_versao4.pdf.

Ferreira, E. A., Alves, D. C. S. Q., Parnaíba, F. J. B., da Silva, N. D., Barreto, S. C., Rodrigues, A. C. S., ... \& Marcelino, S. A. (2019). Fatores Associados a não Adesão ao Tratamento da Hipertensão Arterial Sistêmica em Clientes Idosos/Factors Associated with Non-Adherence to the Treatment of Systemic Arterial Hypertension in Elderly. ID on line REVISTA DE PSICOLOGIA, 13(44), 865-876. https://doi.org/10.14295/idonline.v13i44.1660.

Kretchy, I. A., Asiedu-Danso, M., \& Kretchy, J. P. (2021). Medication management and adherence during the COVID-19 pandemic: perspectives and experiences from low-and middle-income countries. Research in social and administrative pharmacy, 17(1), 2023-2026. https://www.ncbi.nlm.nih.gov/pmc/articles/PMC7158799/.

Le, T.; Toscani, M.; Colaizzi, J. (2020). Telefarmácia: um novo paradigma para nossa profissão. Journal of Pharmacy Practice, 33(2), 176-182. https://pubmed.ncbi.nlm.nih.gov/30060679/.

Liu, S., Luo, P., Tang, M., Hu, Q., Polidoro, J. P., Sun, S., \& Gong, Z. (2020). Providing pharmacy services during the coronavirus pandemic. International journal of clinical pharmacy, 42(2), 299-304. https://onlinelibrary.wiley.com/doi/full/10.1111/ijcp.13890.

Lythgoe, M. P., \& Middleton, P. (2020). Ongoing clinical trials for the management of the COVID-19 pandemic. Trends in pharmacological sciences, 41(6), 363-382. https://www.sciencedirect.com/science/article/abs/pii/S0165614720300705.

McKee, D. L., Sternberg, A., Stange, U., Laufer, S., \& Naujokat, C. (2020). Candidate drugs against SARS-CoV-2 and COVID-19. Pharmacological research, 157, 104859. https://doi.org/10.1016/j.phrs.2020.104859.

Mehra, M. R., Desai, S. S., Ruschitzka, F., \& Patel, A. N. (2020). RETRACTED: Hydroxychloroquine or chloroquine with or without a macrolide for treatment of COVID-19: a multinational registry analysis. 2020. https://doi.org/10.1016/S0140-6736(20)31180-6. 
Research, Society and Development, v. 10, n. 12, e230101220287, 2021

Mendonça, R. O. L.; Rossoni, H. A. V. (2019). Logística Reversa de Medicamentos: Oportunidades para o farmacêutico, benefício para o meio ambiente. Cartilha Reserva de medicamentos - CRFMG. https://www.crfmg.org.br/site/uploads/areaTecnica/20200117[150411]Cartilha_de_ Logistica_Reversa_de_Medicamentos.pdf.

Oliveira, N. V. B. V. D., Szabo, I., Bastos, L. L., \& Paiva, S. P. (2017). Atuação profissional dos farmacêuticos no Brasil: perfil sociodemográfico e dinâmica de trabalho em farmácias e drogarias privadas. Saúde e Sociedade, 26, 1105-1121. http://dx.doi.org/10.1590/s0104-12902017000002.

Resende, L. C. (2016). Implantação do serviço de acompanhamento farmacoterapêutico em unidade de atenção primária à saúde de Betim: experiência do Projeto Dia a Dia. Experiências Exitosas de Farmacêuticos no SUS, 4(4), 40-48. http://revistas.cff.org.br/?journal= experienciasexitosas\&page $=$ article $\&$ op $=$ view $\&$ path $\% 5 \mathrm{~B} \% 5 \mathrm{D}=1462$

Rossignol, J. F. (2016). Nitazoxanide, a new drug candidate for the treatment of Middle East respiratory syndrome coronavirus. Journal of infection and public health, 9(3), 227-230. https://doi.org/10.1016/j.jiph.2016.04.001.

Rossignoli, P., Pontarolli, D. R. S., Moretoni, C. B., Limberger, P. M., \& Nunes, M. (2020). Enfrentamento da Covid-19 nas unidades de assistência farmacêutica na Secretaria de Saúde do Paraná. Revista de Saúde Pública do Paraná, 3(Supl.). http://www.saude.pr.gov.br.

Silveira, M. C. D. (2019). O uso Off Label de Medicamentos no Brasil (Doctoral dissertation). https://www.arca.fiocruz.br/handle/icict/39683.

Song, Z., Hu, Y., Zheng, S., Yang, L., \& Zhao, R. (2021). Hospital pharmacists' pharmaceutical care for hospitalized patients with COVID-19: Recommendations and guidance from clinical experience. Research in Social and Administrative Pharmacy, 17(1), 2027-2031. https://pubmed.ncbi.nlm.nih.gov/32273253/.

Tay, M. Z., Poh, C. M., Rénia, L., MacAry, P. A., \& Ng, L. F. (2020). The trinity of COVID-19: immunity, inflammation and intervention. Nature Reviews Immunology, 20(6), 363-374. https://www.nature.com/articles/s41577-020-0311-8.

Torres, P. L., Messias, M., do Nascimento, S. S., Tsoulfas, V. T. M., Rezende, E., \& de Aguiar, V. L. (2020). Guia de acolhimento com classificação de risco e avaliação de vulnerabilidades para enfermeiros da atenção básica. Editora dos Editores. http://revista.farmacoterapia.pt/index.php/rpf/article/view/279.

Touret, F., \& de Lamballerie, X. (2020). Of chloroquine and COVID-19. Antiviral research, 177, 104762. https://pubmed.ncbi.nlm.nih.gov/32147496/.

UNICEF. (2020). ORGANIZAÇÃO PAN-AMERICANA DA SAÚDE; ORGANIZAÇÃO MUNDIAL DA SAÚDE. Mensagens e ações importantes para a COVID-19 prevenção e controle em escolas. OPAS/BRA/Covid-19/20-015. Brazil. https://www.paho.org/bra/.

Yavuz, S., \& Ünal, S. (2020). Antiviral treatment of COVID-19. Turkish journal of medical sciences, 50(SI-1), 611-619. https://pubmed.ncbi.nlm.nih.gov/32293834/.

Ying, W., Qian, Y., \& Kun, Z. (2021). Drugs supply and pharmaceutical care management practices at a designated hospital during the COVID-19 epidemic. Research in Social and Administrative Pharmacy, 17(1), 1978-1983. https://www.ncbi.nlm.nih.gov/pmc/articles/PMC7136878/.

Zheng, Y. Y., Ma, Y. T., Zhang, J. Y., \& Xie, X. (2020). COVID-19 and the cardiovascular system. Nature Reviews Cardiology, 17(5), 259-260. https://www.nature.com/articles/s41569-020-0360-5

Zheng, S. Q., Yang, L., Zhou, P. X., Li, H. B., Liu, F., \& Zhao, R. S. (2021). Recommendations and guidance for providing pharmaceutical care services during COVID-19 pandemic: a China perspective. Research in social and administrative pharmacy, 17(1), 1819-1824. https://pubmed.ncbi.nlm.nih.gov/32249102/. 